\title{
Formal Modelling of Service-Oriented Systems
}

\author{
Antonia Lopes \\ Department of Informatics, \\ Faculty of Sciences, University of Lisbon, \\ Portugal \\ mal@di.fc.ul.pt
}

\begin{abstract}
In service-oriented systems interactions are no longer based on fixed or programmed exchanges between specific parties but on the provisioning of services by external providers that are procured on the fly subject to a negotiation of service level agreements (SLAs).

This research addresses the challenge raised on software engineering methodology by the need of declaring such requirements as part of the models of service-oriented applications, reflecting the business context in which services and activities are designed.

In this talk, we report on the formal approach to service-oriented modelling that we have developed which aimed at providing formal support for modelling service-oriented systems in a way that is independent of the languages in which services are programmed and the platforms over which they run. We discuss the semantic primitives that are being provided in SRML (SENSORIA Reference Modelling Language) for modelling composite services, i.e. services whose business logic involves a number of interactions among more elementary service components as well the invocation of services provided by external parties. This includes a logic for specifying stateful, conversational interactions, a language and semantic model for the orchestration of such interactions, and an algebraic framework supporting service discovery, selection and dynamic assembly.
\end{abstract}

